\title{
Bit Loading With BER-Constraint for Multicarrier Systems
}

\author{
Alexander M. Wyglinski, Student Member, IEEE, Fabrice Labeau, Member, IEEE, and Peter Kabal, Member, IEEE
}

\begin{abstract}
We present discrete adaptive bit loading algorithms for multicarrier systems with uniform (nonadaptive) power allocation operating in a frequency selective fading environment. The algorithms try to maximize the overall throughput of the system while guaranteeing that the mean bit error rate (BER) remains below a prescribed threshold. We also study the impact of imperfect subcarrier signal-to-noise ratio information on throughput performance. Results show that the proposed algorithms have approximately the same throughput and mean BER as the optimal allocation while having a significantly lower computational complexity relative to other algorithms with near-optimal allocations. Moreover, when compared with algorithms that employ approximations to water filling, the computational complexity is comparable while the overall throughput is closer to the optimum.
\end{abstract}

Index Terms-Adaptive modulation, frequency selective channel, multicarrier modulation, wireless LAN.

\section{INTRODUCTION}

M ULTICARRIER modulation is the method of choice for many data transmission systems, including wireless local area networks (WLAN) applications [1], [2]. In conventional wireless orthogonal frequency division multiplexing systems, all subcarriers employ the same signal constellation. However, the overall error probability is dominated by the subcarriers with the worst performance. To improve performance, adaptive bit and power allocation (i.e., "loading") algorithms can be employed, where the signal constellation size and power distribution vary according to the measured signal-to-noise ratio (SNR) values across the subcarriers. In extreme situations, some subcarriers can be "turned off" or nulled if the subcarrier SNR values are poor.

Most published proposals of bit and power allocation algorithms are variants of three basic types of algorithms: incremental (i.e., "greedy") allocation [3], [4], bit loading based on channel capacity approximations [5], [6], and bit loading based on probability of bit error expressions [7], [8]. The first type incrementally allocates an integer number of bits at the cost of high computational complexity. The other two types of algorithms use closed-form expressions of performance measures in order to determine a bit allocation but require rounding to integer values, which may lead to allocations that are far from the optimum. Therefore, the implementation of loading

Manuscript received July 29, 2003; revised December 4, 2003; accepted May 10, 2004. The editor coordinating the review of this paper and approving it for publication is D. Gesbert. This research was supported in part by the Natural Sciences and Engineering Research Council of Canada and Le Fonds de Recherche sur la Nature et les Technologies du Québec.

A. M. Wyglinski was with the Department of Electrical and Computer Engineering, McGill University, Montréal, QC, Canada. He is now with the Information and Telecommunication Technology Center, The University of Kansas, Lawrence, KS 66045 USA (e-mail: alexw@ieee.org).

F. Labeau and P. Kabal are with the Department of Electrical and Computer Engineering, McGill University, Montréal, QC H3A 2T5, Canada.

Digital Object Identifier 10.1109/TWC.2005.850313 algorithms is usually a tradeoff between how close they come to the optimum allocation and how quickly they reach their final allocation.

Common choices for objective functions that loading algorithms are attempting to optimize are the maximization of the overall throughput given a total power constraint, known as rate-adaptive loading, and the minimization of the energy given a fixed throughput, known as margin-adaptive loading [5].

In this work, we propose two discrete rate-adaptive loading algorithms that try to balance the implementation tradeoffs while coming close to the maximum throughput. The details of these algorithms are presented in Sections II and III. As in other studies, we consider only uncoded systems for the sake of straightforward comparison. However, the introduction of coding would improve the performance relative to an uncoded system and can be accounted for by a nonlinear modification of the SNR values in relationship to the coding gain. ${ }^{1}$ Many adaptive allocation algorithms can perform both adaptive bit and power loading; the algorithms studied and evaluated in this paper employ only adaptive bit loading and uniform power allocation. ${ }^{2}$

\section{Proposed Incremental Allocation}

The adaptive bit loading algorithms proposed in this paper try to solve

$$
\max _{b_{i}} \sum_{i=1}^{N} b_{i}, \quad \text { subject to } \quad \bar{P}=\frac{\sum_{i=1}^{N} b_{i} P_{i}}{\sum_{i=1}^{N} b_{i}} \leq P_{\mathrm{T}}
$$

where $b_{i}$ is the number of bits for subcarrier $i, \bar{P}$ is the mean bit error rate (BER), $P_{\mathrm{T}}$ is the specified BER threshold, and $P_{i}$ is the BER for subcarrier $i$, which is determined from the subcarrier SNR value $\gamma_{i}$. As in other studies [5]-[9], these SNR values are assumed to be known at both the transmitter and the receiver using data-assisted channel estimation techniques.

To compute the probability of bit error, closed-form expressions of all the modulation schemes that can be employed by the system [binary phase-shift keying (BPSK), quaternary phase-shift keying (QPSK), rectangular 16-quadraticamplitude modulation (QAM), and 4-QAM] are used. For instance, the probability of bit error for BPSK is given by [11]

$$
P_{2, i}\left(\gamma_{i}\right)=Q\left(\sqrt{2 \gamma_{i}}\right)
$$

\footnotetext{
${ }^{1}$ In general, the overall gain of using both coding and adaptive modulation is less than the sum of the individual gains, as they both exploit the same sources of diversity.

${ }^{2}$ Although power allocation can provide substantial gains, it has been shown [9], [10] that, e.g., for WLANs, the regulatory requirements do not permit exploitation of power reallocation to any great extent.
} 
while the probability of symbol error for QPSK $\left(M_{i}=4\right)$, rectangular 16-QAM $\left(M_{i}=16\right)$, and rectangular 64-QAM $\left(M_{i}=64\right)$ is given by [11]

$$
\begin{aligned}
P_{M_{i}, i}\left(\gamma_{i}\right)=4(1 & \left.-\frac{1}{\sqrt{M_{i}}}\right) Q\left(\sqrt{\frac{3 \gamma_{i}}{M_{i}-1}}\right) \\
\cdot & {\left[1-\left(1-\frac{1}{\sqrt{M_{i}}}\right) Q\left(\sqrt{\frac{3 \gamma_{i}}{M_{i}-1}}\right)\right] }
\end{aligned}
$$

from which the probability of bit error is obtained using the approximation $P_{i} \approx P_{M_{i}, i} / \log _{2}\left(M_{i}\right)$.

Using an incremental bit allocation algorithm, the signal constellation configuration for the subcarriers can be determined via the following algorithm.

1) Initialization: set the modulation scheme of all the subcarriers to 64-QAM.

2) Determine $P_{i}, i=1, \ldots, N$, given the subcarrier SNR values, using (2) or (3).

3) Compare $\bar{P}$ with $P_{\mathrm{T}}$. If $\bar{P}$ is less than $P_{\mathrm{T}}$, the current configuration is kept and the algorithm ends.

4) Search for the subcarrier with the worst $P_{i}$ and reduce the constellation size. If $b_{i}=1$, null the subcarrier (i.e., set $\left.b_{i}=0\right)$.

5) Recompute $P_{i}$ of all subcarriers with changed allocations and return to Step 3.

Although this bit loading algorithm does not perform power allocation, it can be easily modified to include it [9], [10]. Furthermore, optimality can only be guaranteed if the algorithm achieves $\bar{P} \leq P_{\mathrm{T}}$ while removing the fewest number of bits. For example, suppose the two subcarriers with the worst BER $i$ and $j$ employ 64-QAM and QPSK modulation, respectively. Decreasing the signal constellation size of either subcarrier will result in $\bar{P} \leq P_{\mathrm{T}}$. If subcarrier $i$ is chosen, the allocation is not optimal since it is reduced by 2 bits per symbol epoch while subcarrier $j$ is reduced by just 1 bit per symbol epoch. ${ }^{3}$

\section{Proposed Peak BER-Constrained Allocation}

\section{A. Bit Allocation}

Although the first proposed bit allocation algorithm usually attains near-optimal solutions, its computational complexity is still rather high at low SNR values (see Section VI). What is needed is an algorithm that accurately determines the final bit allocation in an iterative low computational complexity fashion.

It is straightforward to allocate bits to each subcarrier so that the subcarrier BER $P_{i}$ is below some peak BER constraint $\hat{P}$ : we first have to evaluate $P_{M_{i}, i}$ for all possible $i$ and $M_{i}$, and pick for each subcarrier the constellation size $M_{i}=2^{b_{i}}$ that is maximum while still having $P_{M_{i}, i} \leq \hat{P}$. We then choose to use this peak BER constraint $\hat{P}$ as a proxy to satisfy an average BER constraint $\bar{P}$. A first guess on $\hat{P}$ is taken, the bits $b_{i}$ are allocated accordingly, and the resulting $\bar{P}$ is computed. If $\bar{P}$ is below (respectively above) $P_{\mathrm{T}}, \hat{P}$ is increased (respectively

\footnotetext{
${ }^{3}$ When going from 64-QAM to 16-QAM or 16-QAM to QPSK, the reduction in bits per symbol epoch is 2, while for QPSK to BPSK or BPSK to null, the reduction is 1
}

decreased) by an amount $\delta$ in the logarithmic domain at every iteration. The value of $\hat{P}$ is adjusted in this way until $\bar{P}$ exceeds (respectively goes below) $P_{\mathrm{T}}$, in which case $\delta$ is reduced.

The complete operation of the proposed algorithm is described as follows.

1a) Calculate $\bar{P}$ for the case when all subcarriers employ the largest signal constellation.

1b) If the resulting $\bar{P}$ is below $P_{\mathrm{T}}$, set the final allocation to the largest signal constellation for all subcarriers and end the algorithm. ${ }^{4}$

1c) Calculate $P_{i}$ for all the subcarriers employing the smallest nonnulled signal constellation.

1d) If the smallest $P_{i}$ is above $P_{\mathrm{T}}, P_{\mathrm{T}}$ cannot be achieved; end the algorithm.

2) Find the largest signal constellation for each subcarrier for which $P_{i}$ is below $\hat{P}$.

3) Compute the current value of $\bar{P}$.

4) If the current and previous values of $\bar{P}$ are either both above or both below $P_{\mathrm{T}}$, go to Step 5, else go to Step 6 .

5) If both current and previous $\bar{P}$ values are above $P_{\mathrm{T}}$, reduce $\hat{P}$ by a factor $\delta$ and go to Step 2, else increase $\hat{P}$ by a factor $\delta$ and go to Step 2 .

6) If the previous and current allocations differ by one signal constellation level, make the allocation with $\bar{P}$ below $P_{\mathrm{T}}$ the final allocation and end the algorithm, else go to Step 7.5

7) Reduce $\delta$ by half.

8) If the current allocation gives a $\bar{P}$ that is above $P_{\mathrm{T}}$, reduce $\hat{P}$ by a factor $\delta$ and go to Step 2, else increase $\hat{P}$ by a factor $\delta$ and go to Step 2 .

\section{B. Initial Peak BER Threshold Calculation}

The speed at which the algorithm in Section III-A reaches its final allocation depends on the choice of the initial $\hat{P}$ and the $\delta$ it uses. This section describes how to estimate the initial values for $\hat{P}$ and $\delta$ using the subcarrier SNR values.

One approach to this problem is to determine how much any given subcarrier can individually exceed $P_{\mathrm{T}}$ while $\bar{P}$ remains below it. Given that a subcarrier can support $B$ possible modulation schemes, resulting in $B$ possible values for $P_{i}$, we define the largest $P_{i}$ value that is below $P_{\mathrm{T}}$ as $\beta_{i}$ and the smallest value of $P_{i}$ above $P_{\mathrm{T}}$ as $\alpha_{i}$. Therefore, knowing that the mean of $\beta_{i}$ is below $P_{\mathrm{T}}$, we incrementally replace the smallest $\beta_{i}$ with the corresponding $\alpha_{i}$ until $\bar{P}>P_{\mathrm{T}}$.

The algorithm for finding the initial peak BER $\hat{P}$ estimate is as follows.

1) Given the subcarrier SNR values $\gamma_{i}$, calculate $P_{i}$ for all the different modulation schemes that could potentially be employed in the system.

2) Find $\beta_{i}$, the largest $P_{i}$ that does not exceed $P_{\mathrm{T}}$.

3) Find $\alpha_{i}$, the smallest $P_{i}$ that exceeds $P_{\mathrm{T}}$.

\footnotetext{
${ }^{4}$ This provides for a quick exit from the algorithm when the subcarrier SNR values are large enough to have the system operate at maximum throughput.

${ }^{5}$ If the previous and current $\bar{P}$ values straddle $P_{\mathrm{T}}$ as well as differ by one signal constellation, it is obvious that the additional bit(s) is (are) the cause of the violation of the mean BER constraint.
} 
4) Find all values of $\beta_{i}$ that are within an order of magnitude of $\max _{i} \beta_{i}$ and assign their indices to a set $\mathcal{S}\left(\beta_{i}\right.$ not within an order of magnitude can be neglected).

5) Given $\beta_{i}, i \in \mathcal{S}$, we need to solve for $\Delta P$ given

$$
\Delta P=\sum_{i \in \mathcal{S}} b_{i}\left(P_{\mathrm{T}}-\beta_{i}\right)
$$

in order to determine by how much several subcarriers can violate the condition $P_{i} \leq P_{\mathrm{T}}$ while the system still satisfies $\bar{P} \leq P_{\mathrm{T}}$.

6) Sort the values of $\alpha_{i}$ in increasing order. Find the largest value of $I$ for which

$$
\Delta P \geq \sum_{i=0}^{I} b_{i}\left(\alpha_{i}-P_{\mathrm{T}}\right)
$$

is true, where $0 \leq I<N$, and set $\alpha_{I}$ as the initial $\hat{P}$ for the algorithm described in Section III-A.

The initial value of $\delta$ is proportional to the average SNR of the system $\bar{\gamma}$. It has been observed in several simulations that for low $\bar{\gamma}$ values, small values for $\delta$ resulted in the algorithm converging quickly to a final solution, while for high $\bar{\gamma}$ values, large values of $\delta$ resulted in quickly obtaining the solution. Thus, choosing the values for $\delta$ between the two extremes, $\delta$ decreases linearly as a function of $\bar{\gamma}$.

Using these values of $\delta$ in conjunction with the $\hat{P}$ "smart" initialization algorithm, the number of iterations required to find the final $\hat{P}$ can be reduced by as much as a factor of two when compared to a scheme using a fixed initialization.

\section{BIT LOAding With IMPERFECT SUbCARRIER SNR INFORMATION}

Although many studies on adaptive bit loading algorithms make the assumption that the subcarrier SNR values are perfectly known, this is not the case in reality. Therefore, it is necessary to investigate the impact of imperfect subcarrier SNR information on the throughput of these systems. In particular, two sources of error in subcarrier SNR information are studied: 1) channel estimation error and 2) quantization error.

\section{A. Models for Imperfect SNR Information}

1) Gaussian Subcarrier SNR Noise Model: Channel estimation in multicarrier systems, especially WLAN systems [1], uses predefined training symbols across the subcarriers intermittently to extract channel characteristics. From these characteristics, subcarrier SNR values are computed and used by the adaptive bit loading algorithms.

The errors accompanying the channel estimates also get translated into subcarrier SNR values, resulting in a corrupted SNR value for subcarrier $i$. This can be modeled by $\hat{\gamma}_{i}=$ $\gamma_{i}+\epsilon_{i}$, where $\gamma_{i}$ is the actual SNR value for subcarrier $i$ and $\epsilon_{i}$ is the error due to the channel estimation process. A similar expression was used by Leke and Cioffi [12].

In this work, we assume that $\epsilon_{i}$ has a normal distribution with zero mean and variance $\sigma^{2}$. However, it is essential to avoid a negative $\hat{\gamma}_{i}$. Therefore, when $\gamma_{i}+\epsilon_{i}<0$ occurs, we set $\hat{\gamma}_{i}=0$.
2) SNR Quantization Noise Model: Since the adaptive bit loading algorithms require the translation of subcarrier SNR values into $P_{i}$ values, a look-up table is employed. However, this implies that the subcarrier SNR values must be quantized. ${ }^{6}$

We must determine where to place the quantizer reproduction levels $d_{k}$ in order to minimize the quantization error for all the modulation schemes. Since we want adequate resolution of the BER waterfall curves around $P_{\mathrm{T}}$, the output levels should be concentrated at that point. Given $q$ bits to represent a quantizer reproduction level, the number of levels is defined as $2^{q}$. The following algorithm tries to achieve this through a suboptimal placement of $d_{k}$.

1) Determine the pair of SNR values to obtain the probability of bit error values $P_{i}$ that are two orders of magnitude above and below $P_{\mathrm{T}}$ for each modulation scheme, thus forming regions $Q_{k}$, for $k=1, \ldots, B$, where $B$ is the number of modulation schemes.

2) For the $B$ modulation schemes, put $2^{q} / B$ output levels uniformly in $Q_{k}$ for all $k$. In the case of overlapping regions, combine them and their allocation of output levels, distributing the levels uniformly across the combined region.

By distributing $d_{k}, k=0, \ldots, 2^{q}-1$, in this way, the BER waterfall curves are ensured of quantization with enough resolution.

\section{Simulation Setup}

The IEEE Std. 802.11a [1], a WLAN standard that employs conventional multicarrier modulation, is referred to for realistic operating parameters. The system employs the proposed loading algorithms based on the parameters of the standard system. ${ }^{7}$ However, unlike the standard, where the same modulation scheme is employed across all the subcarriers, the proposed algorithms can use a different modulation scheme for each subcarrier. In addition, subcarriers can be turned off. Multicarrier systems operating at $P_{\mathrm{T}}=10^{-3}$ and $10^{-5}$, employing the optimization algorithm of Fox $[3],{ }^{8}$ the algorithm of Leke and Cioffi [5], ${ }^{9}$ and the two proposed algorithms, were studied. ${ }^{10}$ Furthermore, an exhaustive search algorithm to find the optimal bit allocation was also employed for the subcarriers over a portion of the band (to keep the complexity manageable).

${ }^{6} \mathrm{SNR}$ estimation is performed at the receiver while bit allocation is normally performed at the transmitter. Therefore, a feedback channel is employed to transmit quantized SNR values.

${ }^{7}$ These parameters are: $N=52$ subcarriers, an operating frequency of $5.15-5.25 \mathrm{GHz}$, a signal bandwidth of $16.6 \mathrm{MHz}$, and the possible modulation schemes are BPSK, QPSK, 16-QAM, and 64-QAM.

${ }^{8}$ With the power allocation set to be uniform across all subcarriers, the algorithm starts with zero bits across all subcarriers as an initial allocation and allocates to subcarrier $i$ for which $\Delta b_{i} / \Delta P_{i}$ is a maximum. The incremental allocation continues until $\bar{P}>P_{\mathrm{T}}$.

${ }^{9}$ To obtain a constant uniform power allocation across all subcarriers, the expression for the noise-to-signal ratio (NSR) in [5] was modified to $\mathrm{NSR}=\left(\epsilon_{\mathrm{sc}} / \Gamma\right)+\left(1 / N_{\mathrm{on}}\right) \sum^{N_{\mathrm{on}}}\left(1 / g_{n}\right)$, where $\epsilon_{\mathrm{Sc}}$ is the subcarrier power (a constant value across all subcarriers), $N_{\mathrm{O}}$ is the number of subcarriers that are "on", and $g_{n}$ is the SNR of subchannel $n$.

${ }^{10} \mathrm{We}$ have not implemented any probability of error-based algorithms since the bit loading component of the probability of error-based algorithms is heavily dependent on adaptive power allocation. Furthermore, any bit allocations would have to be rounded to integer values, thus adversely affecting the performance. 


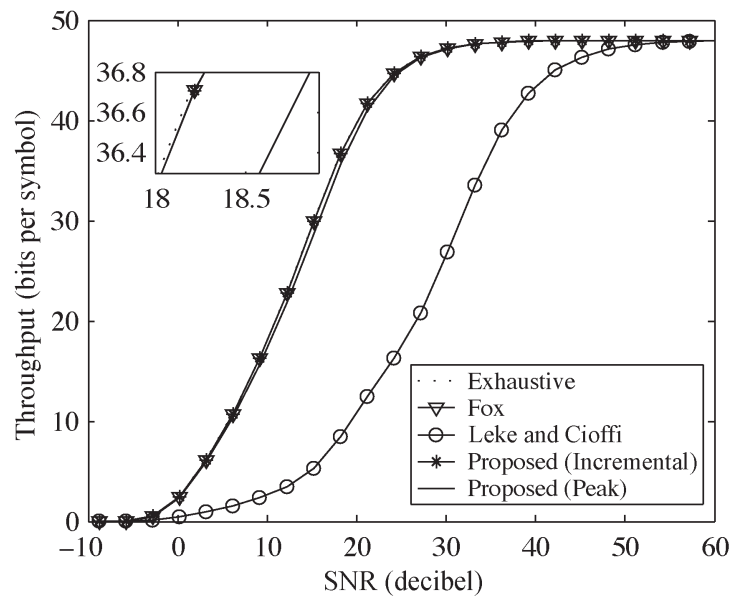

Fig. 1. Throughput results for an eight-subcarrier system employing an exhaustive bit allocation algorithm (no marker, dotted line), the optimization algorithm of Fox [3] (triangular marker, solid line), the algorithm of Leke and Cioffi [5] (circular marker, solid line), the proposed incremental algorithm (asterisk marker, solid line), and the proposed peak BER algorithm (no marker, solid line) given a $P_{\mathrm{T}}$ of $10^{-3}$. Note how close the results are for all but one of the algorithms.

The statistical indoor propagation modeling technique employing a Rayleigh fading statistic due to Saleh and Valenzuela [13] was used. We used a mean cluster arrival time of $100 \mu \mathrm{s}$, a mean ray arrival time of $1 \mu \mathrm{s}$, a cluster power-decay time constant of $20 \mu \mathrm{s}$, and a ray power-decay time constant of $6 \mu \mathrm{s}$. For each time-invariant channel realization, the algorithms were operated at 70 different average SNR values ranging from -11 to $59 \mathrm{~dB}$. The trials were repeated for 10000 different channel realizations and the results averaged.

\section{Simulation Results}

In Fig. 1, the bit allocation algorithms are compared for the case of eight subcarriers and $P_{\mathrm{T}}=10^{-3}$. All the algorithms, except one, approach the optimal throughput results (determined using exhaustive search). The algorithm of Leke and Cioffi does not reach the same throughput as the other systems until high SNR values of $49 \mathrm{~dB}$. Moreover, since Leke and Cioffi's algorithm does not check if the bit allocation exceeds $P_{\mathrm{T}}$, there is a possibility that $P_{\mathrm{T}}$ may be violated. ${ }^{11}$ Thus, those allocations were not included in the results since they would result in throughputs that are greater than the maximum possible throughput given $\bar{P} \leq P_{\mathrm{T}}$.

When 52 subcarriers are employed for a $P_{\mathrm{T}}=10^{-5}$, as shown in Fig. 2, the algorithms achieve nearly the same throughput. When noisy subcarrier SNR values on throughput performance are employed by the algorithms, the throughput of the system decreases as the variance increases, except for Leke and Cioff's algorithm, which is already far from the optimal allocation. In Fig. 3, quantized subcarrier SNR values are employed. An increase in the quantizer output levels results

\footnotetext{
${ }^{11}$ At SNR values of 0,5 , and $10 \mathrm{~dB}$, the number of violations of $P_{\mathrm{T}}$ as a percentage of the total number of channel realizations was $8.23,3.53$, and $9.66 \%$ for eight subcarriers and $P_{\mathrm{T}}=10^{-3}$. For 52 subcarriers and $P_{\mathrm{T}}=$ $10^{-5}$, given the same SNR values, these percentages are 54.95, 96.84, and $99.94 \%$
}

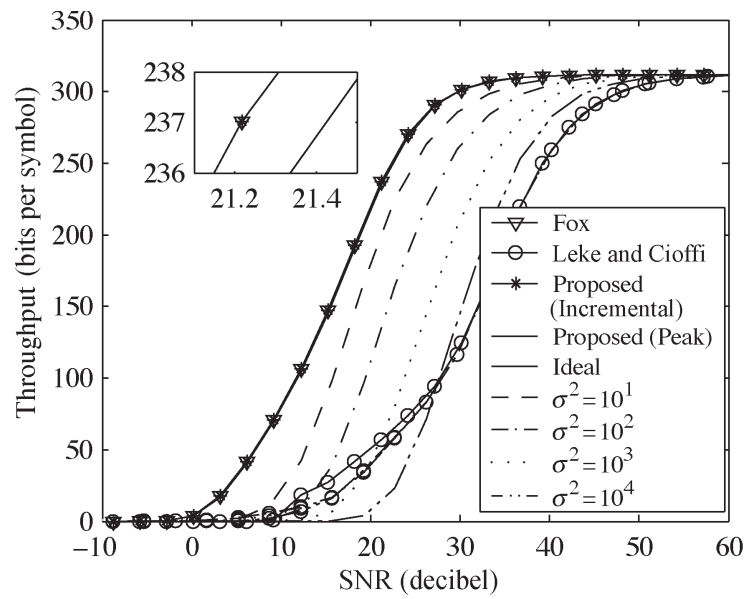

Fig. 2. Throughput results for a 52-subcarrier system employing the optimization algorithm of Fox [3] (triangular marker), the algorithm of Leke and Cioffi [5] (circular marker), the proposed incremental algorithm (asterisk marker), and the proposed peak BER algorithm (no marker) given a $P_{\mathrm{T}}$ of $10^{-5}$ and different amounts of Gaussian noise added to the known subcarrier SNR values. Results for the following cases were obtained: no noise added (solid line), $\sigma^{2}=10^{1}$ (dashed line), $\sigma^{2}=10^{2}$ (dash-dotted line), $\sigma^{2}=10^{3}$ (dotted line), and $\sigma^{2}=10^{4}$ (dash-dot-dotted line). Note that for Fox and the proposed incremental algorithm, only the "no noise" results are presented.

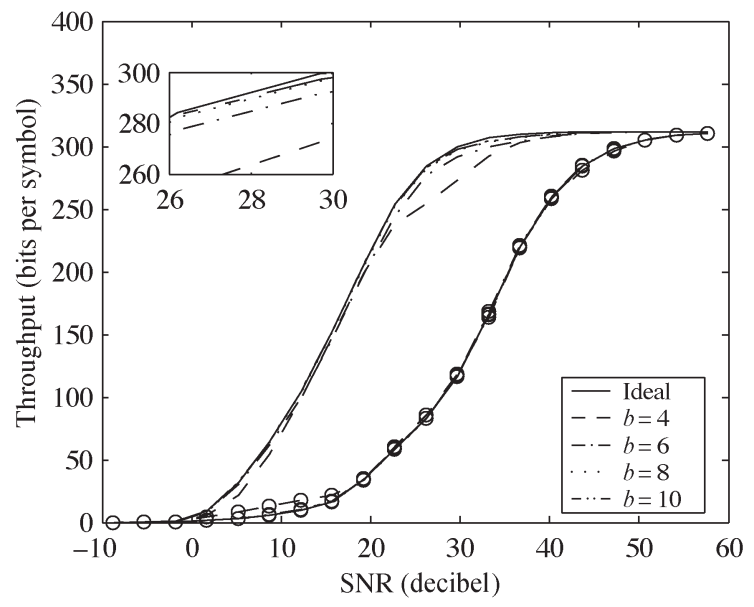

Fig. 3. Throughput results for a 52-subcarrier system employing the proposed peak BER algorithm (no circles) and the algorithm of Leke and Cioffi [5] (with circles) given a $P_{\mathrm{T}}=10^{-5}$. The subcarrier SNR values are quantized with $2^{b}$ levels for the following cases: no quantization (solid line), $b=4$ (dashed line), $b=6$ (dash-dotted line), $b=8$ (dotted line), and $b=10$ (dash-dotdotted line). Note that the latter uses another set of quantization reproduction levels.

in a decrease of granular error that corresponds to improved throughput performance.

Since most of the algorithms are close to the maximum achievable throughput given the maximum error constraint, the addition of Gaussian or quantization noise to the subcarrier SNR values can cause the system to either violate the constraint (when $\hat{\gamma}_{i}>\gamma_{i}$ ) or decrease in throughput (when $\hat{\gamma}_{i} \leq \gamma_{i}$ ). Since we are working under the assumption that $\bar{P}>P_{\mathrm{T}}$ is not acceptable, when the former occurs, we record the number of times this occurs (see Fig. 4). Moreover, these allocations are not considered in the throughput results. In practice, an error margin would be employed by the algorithm such that only a small fraction of cases would violate $\bar{P} \leq P_{\mathrm{T}}$ at the cost of some throughput. 


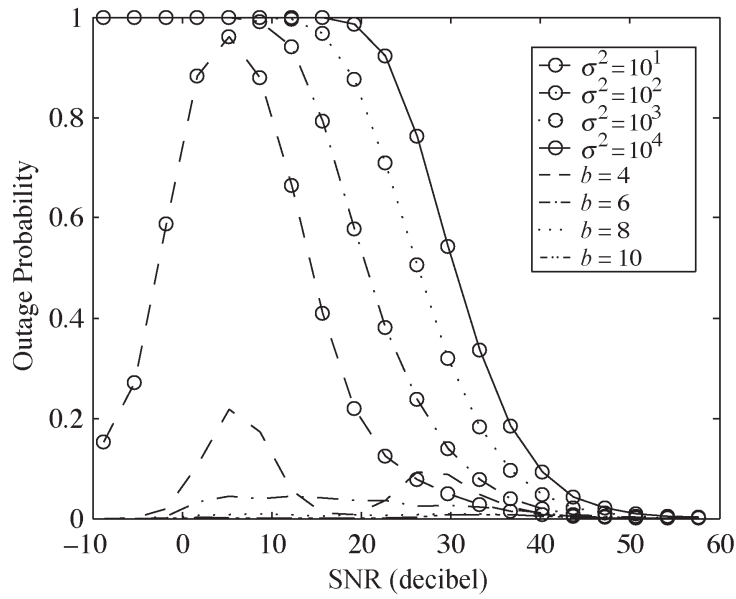

Fig. 4. Outage probability (fraction of realizations for which $\bar{P}>P_{\mathrm{T}}$ ) for a 52-subcarrier system employing the proposed peak BER algorithm at $P_{\mathrm{T}}=$ $10^{-5}$ when (a) Gaussian noise of variance $\sigma^{2}$ is added to the subcarrier SNR values (with circles) and (b) the subcarrier SNR values are quantized with $2^{b}$ levels (without circles).

TABLE I

Mean (Worst) Computation Times in MiLliseconds at DifFERENT SNR VALUES, 52-SUbCARRIERS, $P_{\mathrm{T}}=10^{-5}$ (INTEL PENTIUM IV 2-GHz PROCESSOR)

\begin{tabular}{lcccc}
\hline Algorithm & $10 \mathrm{~dB}$ & $25 \mathrm{~dB}$ & $40 \mathrm{~dB}$ & $55 \mathrm{~dB}$ \\
\hline Fox & $1.13(3.23)$ & $1.48(5.01)$ & $1.41(5.00)$ & $1.37(4.40)$ \\
Leke and Cioffi & $0.94(2.78)$ & $0.96(4.98)$ & $0.93(4.24)$ & $0.90(4.66)$ \\
Proposed (Incremental) & $1.09(2.86)$ & $0.91(4.10)$ & $0.84(2.09)$ & $0.80(2.62)$ \\
Proposed (Peak) & $0.91(2.96)$ & $0.91(2.71)$ & $0.86(3.98)$ & $0.82(4.54)$ \\
\hline
\end{tabular}

A summary of mean and worst case computation times for a 52-subcarrier system with a $P_{\mathrm{T}}$ of $10^{-5}$ is shown in Table I for several SNR values. Furthermore, the cumulative density functions of the computation times at SNR values of 10 and 40 $\mathrm{dB}$ are shown in Fig. 5. For a fair comparison, all algorithms were programmed in $\mathrm{C}$ and executed on the same workstation. It should be noted that although the algorithms may vary in execution time, all the worst case execution times are of the same order of magnitude. This is due to the fact that the worst case computational complexity of all the algorithms under study is of $\mathcal{O}\left(N^{2}\right)$. From these results, the two proposed algorithms achieved near-optimal performance while achieving low computational complexity. Although both perform similarly in terms of throughput and complexity at high SNR values, at low SNR values the proposed peak BER algorithm executes faster than the proposed incremental (both mean and worst cases).

\section{CONCLUSION}

Two new bit allocation algorithms that have high system throughput while guaranteeing a mean BER below a given target value for multicarrier systems are presented. Results showed that both proposed bit allocation algorithms come close to the optimal solution while achieving a low computational complexity. A study of sensitivity to quantization and channel estimation errors has been carried out. When compared to the case of perfect SNR information, the results show that even with

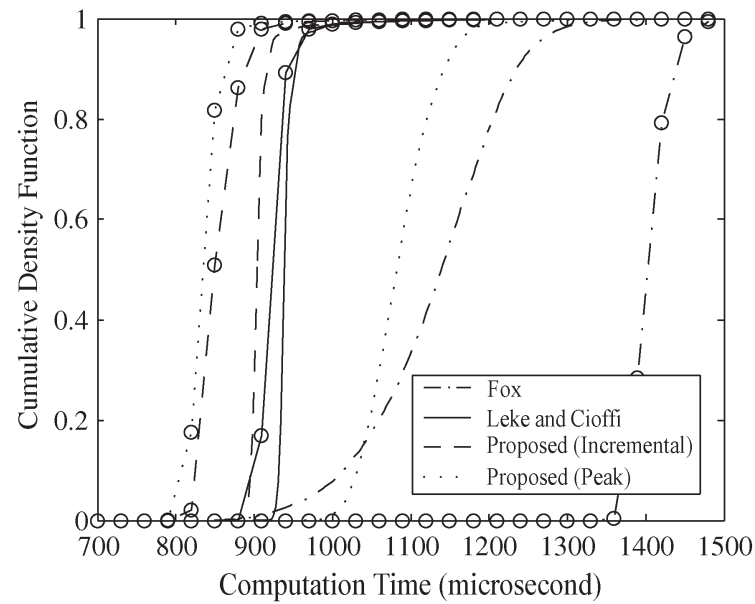

Fig. 5. Cumulative density function of the computational time for a 52 subcarrier system employing the optimization algorithm of Fox [3] (dash-dotted line), the algorithm of Leke and Cioffi [5] (solid line), the proposed incremental algorithm (dotted line), and the proposed peak BER algorithm (dashed line) given a $P_{\mathrm{T}}$ of $10^{-5}$ at SNR values of 10 (without circles) and $40 \mathrm{~dB}$ (with circles). Note that perfectly known subcarrier SNR information was used by the algorithms.

moderate quantization, the bit allocations are closer to optimal. Moreover, depending on how the output levels for the quantizer are positioned, as the number of output levels increases, the quantization error introduced to the SNR information quickly diminishes.

\section{REFERENCES}

[1] Institute of Electrical and Electronics Engineers, Wireless LAN Medium Access Control (MAC) and Physical Layer (PHY) Specifications: HighSpeed Physical Layer in the $5 \mathrm{GHz}$ Band, IEEE Std. 802.11a, Nov. 1999.

[2] European Telecommunications Standards Institute, Broadband Radio Access Networks (BRAN): HIPERLAN Type 2; Physical (PHY) Layer, ETSI TS 101 475, Dec. 2001.

[3] B. Fox, "Discrete optimization via marginal analysis," Manage. Sci., vol. 13, no. 3, pp. 210-216, Nov. 1966.

[4] A. Gersho and R. M. Gray, Vector Quantization and Signal Compression. Boston, MA: Kluwer, 1991.

[5] A. Leke and J. M. Cioffi, "A maximum rate loading algorithm for discrete multitone modulation systems," in Proc. IEEE Global Telecommunications Conf., Phoenix, AZ, 1997, vol. 3, pp. 1514-1518.

[6] J. Campello, "Practical bit loading for DMT," in Proc. IEEE Int. Conf. Communications, Vancouver, Canada, 1999, vol. 2, pp. 801-905.

[7] R. F. H. Fischer and J. B. Huber, "A new loading algorithm for discrete multitone transmission," in Proc. IEEE Global Telecommunications Conf., London, U.K., 1996, vol. 1, pp. 724-728.

[8] I. Kalet, "The multitone channel," IEEE Trans. Commun., vol. 37, no. 2, pp. 119-124, Feb. 1989.

[9] A. M. Wyglinski, P. Kabal, and F. Labeau, "Adaptive filterbank multicarrier wireless systems for indoor environments," in Proc. 56th IEEE Veh. Technol. Conf.-Fall, Vancouver, BC, Canada, 2002, pp. 336-340.

[10] _ - "Adaptive bit and power allocation for indoor wireless multicarrier systems," in Proc. 15th Int. Conf. Wireless Communications, Calgary, AB, Canada, 2003, pp. 500-508.

[11] J. G. Proakis, Digital Communications, 3rd ed. New York: McGrawHill, 1995.

[12] A. Leke and J. M. Cioffi, "Multicarrier systems with imperfect channel knowledge," in Proc. IEEE Int. Symp. Personal, Indoor, Mobile Radio Communications, Boston, MA, 1998, vol. 2, pp. 549-553.

[13] A. A. M. Saleh and R. A. Valenzuela, "A statistical model for indoor multipath propagation," IEEE J. Select. Areas Commun., vol. 5, no. 2, pp. 128-137, Feb. 1987. 